\title{
Surprises in cycling aerodynamics
}

I Bert Blocken ${ }^{1}$, Thijs Defraeye ${ }^{2}$,Erwin Koninckx ${ }^{3}$, Jan Carmeliet ${ }^{4}$ and Peter Hespel ${ }^{5}$ - DOI: 10.1051/epn/2013102

口 ${ }^{1}$ Eindhoven Univ. of Technology - Eindhoven - The Netherlands - b.j.e.blocken@tue.nl

प ${ }^{2}$ Univ. of Leuven - Leuven - Belgium

${ }^{3}$ Flemish Cycling Federation - Brussels - Belgium

口 ${ }^{4}$ Swiss Federal Inst. of Technology Zurich (ETHZ) - Zürich - Switzerland and Swiss Federal Lab. for Materials Testing and Research (Empa) - Dübendorf - Switzerland

口 ${ }^{5}$ Bakala Academy - Athletic Performance Center - Univ. of Leuven - Heverlee, Belgium

Drafting is riding close behind each other to reduce aerodynamic drag. New simulations and measurements for drafting cyclists show that also the leading cyclist experiences a drag reduction, up to $3.1 \%$. For six or more similarly-sized drafting cyclists, the position enjoying the largest drag reduction is the one-but-last position.

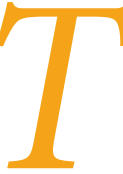

he greatest potential for improvement in cycling speed is aerodynamic" [1]. At racing speeds (about $54 \mathrm{~km} / \mathrm{h}$ or $15 \mathrm{~m} / \mathrm{s}$ in time trails), the aerodynamic resistance or drag is about $90 \%$ of the total resistance [2-5]. Most previously published studies on cycling aerodynamics aimed at reducing the aerodynamic drag of a single cyclist. Fewer publications have addressed the drag reduction due to drafting. In drafting, two or more cyclists ride close behind each other to reduce aerodynamic drag. The few published studies on drafting all confirm the large drag reduction for the trailing riders (up to $30-40 \%$ ), whereas there seems to be a lack of consensus about the effect of drafting on the leading rider. In this respect, Olds [6] stated:

"It has been suggested that riding close behind a leading cyclist will also assist the leading rider in that the low pressure area behind the cyclist will be "filled up" by the trailing rider. However, both Kyle (1979) and McCole et al. (1990) failed to find any measurable effect either in rolldown experiments or in field $\mathrm{VO}_{2}$ measurements."

On the other hand, Computational Fluid Dynamics (CFD) studies on human body models with simplified geometries (such as elliptical cylinders) found drag reductions for the leading cylinder up to 5\% [9]. However, to the best of our knowledge, no studies have yet been published on detailed 3D CFD simulations of drafting cyclists based on realistic human body geometries. Such 3D CFD simulations were the focus of this new study, which included also wind-tunnel measurements. First, the wind-tunnel measurements were used for CFD validation for single cyclists and two drafting cyclists. Next, supported by the validation study, CFD simulations were performed for groups up to eight drafting cyclists, allowing assessment of aerodynamic drag at every position in the group. 


\section{Wind-tunnel measurements}

Three sets of wind-tunnel measurements were done. The first set included overall drag force measurements as well as point measurements with 30 pressure plates on the body of a real cyclist (Cyclist A: height $1.83 \mathrm{~m}$, weight $72 \mathrm{~kg}$ ) in different positions, including the upright position (UP), the dropped position (DP) with straight arms and the timetrial position (TTP) (Figs. 1,2a). The second set comprised overall drag force measurements and point measurements with 115 pressure taps on two reduced-scale (1/2) models of cyclist A, obtained by rapid prototyping (Fig. $2 \mathrm{c}$, d). The third set consisted of overall drag force measurements on two drafting cyclists (Cyclists B and C) behind each other at a wheel-to-wheel separation distance $d=0.15 \mathrm{~m}$. In all measurements, the wheels and legs were static (i.e.,no pedalling). For brevity, only the results of the overall drag force measurements on the real cyclists are presented in this article. A conservative estimate of the measurement error of the drag force is $0.3 \%$ at approach-flow air speed $U_{\infty}=15 \mathrm{~m} / \mathrm{s}$. The measurement results are reported together with the CFD results in the next sections. More detailed information about the wind-tunnel measurements can be found in $[4,5]$.

\section{Computationall models}

Digital models of the cyclist (Cyclist A) were obtained with high-resolution 3D laser scanning, capturing the specific body characteristics in UP,DP and TTP (Fig. 2b). To generate groups of up to eight riders, the cyclist geometry (only cyclist body, not bicycle) was copied and the cyclists were placed behind each other with a wheel-to-wheel separation distance $d=0.01 \mathrm{~m}$. The cyclists were placed in a computational domain with dimensions and spatial discretisation according to best practice guidelines in CFD and based on grid-sensitivity analysis [10-13] (Fig.3).Very small control volumes of $30 \mu \mathrm{m}$ were applied at the cyclist body surface to resolve the boundary layer down to the thin viscous sublayer (Fig. 3b) This is important because boundary layer separation determines to a large extent the aerodynamic drag. Further away from the surface, tetrahedral cells were used with an average size of about $0.03 \mathrm{~m}$. The grids for the single cyclist contained about $7.7 \times 10^{6}$ cells versus $35.6 \times 10^{6}$ cells for the eight drafting cyclists. The simulations were made with a uniform inlet velocity of $15 \mathrm{~m} / \mathrm{s}$ and a turbulence intensity of $0.02 \%$ as in the wind tunnel, representing the relative air movement when cycling at this velocity in still air (zero wind speed). The 3D steady Reynolds-averaged Navier-Stokes (RANS) equations were solved with the standard k- $\varepsilon$ turbulence model [14], near-wall modelling with the one-equation Wolfshtein model [15], pressurevelocity coupling with the SIMPLE algorithm, second-order pressure interpolation and second-order discretisation schemes using the commercial CFD code ANSYS/Fluent 12. Convergence was monitored carefully and the iterations were terminated when all residuals showed no further reduction with increasing number of iterations.

\section{CFD simulations versus wind-tunnel}

\section{measurements}

The CFD simulations only consider the body of the cyclist. Therefore, the corresponding experimental drag area of the cyclist body is obtained by subtracting the experimental drag area of the bicycle configuration plus force platform, which was measured separately, from the total experimental drag area of the cyclist body, bicycle and platform. The deviations between CFD simulations and measurements are 10.5\%,3.5\% and $0.7 \%$ for the UP,DP and TTP, respectively. Given the very low percentage deviation for TTP, it is likely that some errors have cancelled each other.Similar simulations were made for the two drafting cyclists in DP at $d=0.15 \mathrm{~m}$, yielding a drag reduction for the leading cyclist of $1.3 \%$ versus $1.6 \%$ from the wind-tunnel measurements. Both the CFD simulations and the wind-tunnel measurements confirm the drag reduction of the leading rider due to the presence of a trailing rider in his wake. The agreement between the CFD simulations and the wind-tunnel measurements is considered to be very good, which justifies using these simulations for further analysis of the flow field and also using the same computational models (grid, turbulence model, etc.) for the CFD simulations of groups of up to eight drafting cyclists.

\section{Flow-field analysis for two drafting cyclists}

Compared to the single cyclist in TTP, the drag reductions for two drafting cyclists (TTP, $d=0.01 \mathrm{~m}$ ) are $2.6 \%$ for the leading and $13.9 \%$ for the trailing cyclist. The validated CFD simulations are used to explain these drafting effects. Figure 4 displays the pressure coefficient $C_{p}$ in the vertical centre plane and in a horizontal plane at waist height of the cyclist $(\mathrm{s}) . \mathrm{C}_{\mathrm{p}}$ is defined as $\left(P-P_{0}\right) /\left(0.5 \rho U_{\infty}{ }^{2}\right)$ with $P$ the static pressure, $\mathrm{P}_{0}$ the reference static pressure, $\rho$ the air density and $U_{\infty}$ the approach-flow air speed. The figure legend is limited

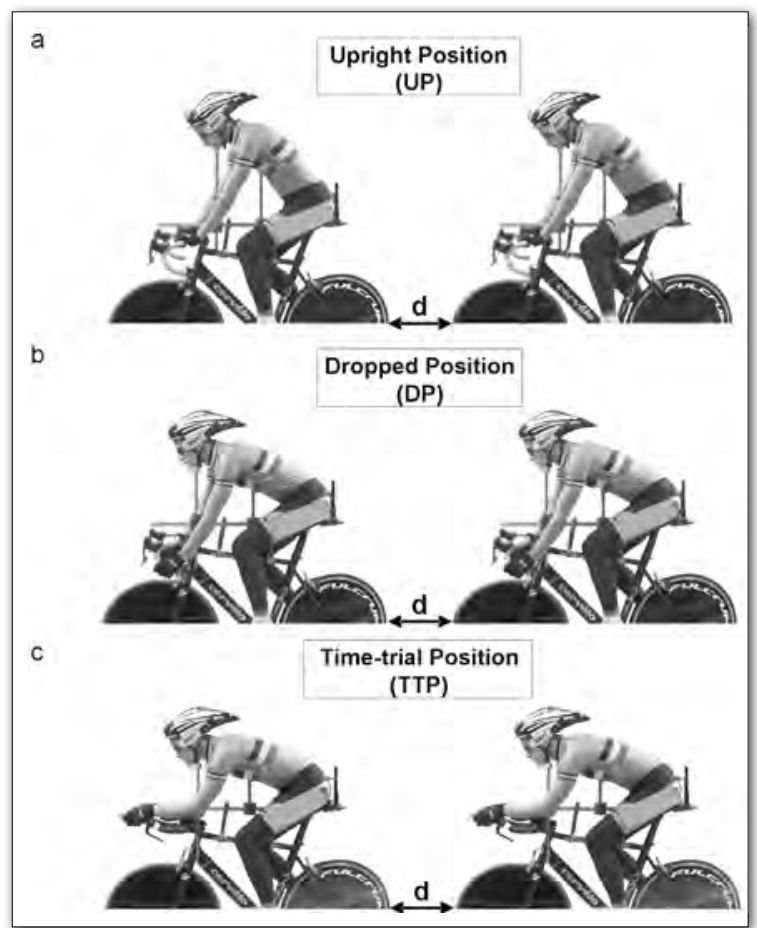

4 FIG. 1:

Three cycling positions with indication of bicycle separation distance d (wheel-to-wheel): (a) Upright position (UP); (b) Dropped position (DP) with straight arms; (c) Time-trial position (TTP), 


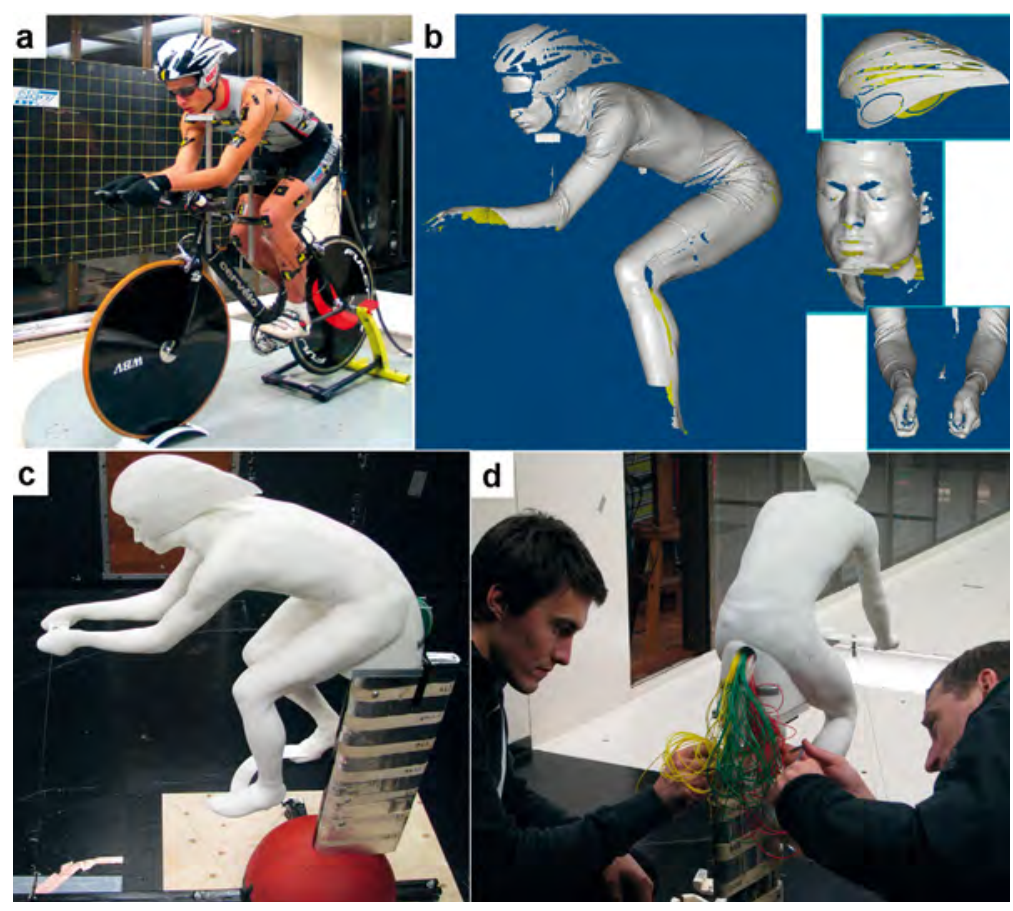

\ FIG. 2:

(a) Cyclist A in the

DNW LST wind tunnel

in Marknesse. The

cyclist and bicycle are positioned on the

force balance and equipped with 30 pressure plates. (b) 3D laser scanning of body geometry of cyclist A. (c) Half-scale model of cyclist A in time-trial position with 115 pressure taps. (d) Halfscale model of cyclist A in upright position with 115 pressure taps. Photo shows pressure tubes exiting from the hollow model. to the interval $[-0.05 ; 0.1]$ in order to highlight the changes in the pressure field due to drafting; the actual maximum and minimum (absolute) values of $C_{p}$ are much larger. The figures clearly show the area of overpressure in front of the cyclists and the area of negative pressure behind them. In case of two drafting cyclists, the wake behind the leading cyclist interacts with the overpressure area in front of the trailing cyclist, which not only results in a drag reduction for the trailing cyclist, but also for the leading cyclist. Note that also the size of the negative pressure area behind the leading rider decreases due to a trailing rider in his wake. This effect shows a striking correspondence with the statement by Olds [6] about the low-pressure area behind the leading cyclist being "filled up" by the trailing cyclist.

\section{Drag reductions for larger groups}

Because the aerodynamic drag of a leading cyclist is significantly reduced by a trailing cyclist in his wake, it can be expected that in larger groups of cyclists, the largest drag reduction is not experienced by the last cyclist - as is generally assumed - but by the one-but-last cyclist. Indeed, while the last cyclist benefits from the leading riders in front of him, the one-but-last cyclist benefits from both the riders in front of him and from the rider behind him. This is confirmed by the CFD simulations: Figure 5 shows the drag reduction for every cyclist in a group of 2,4,6 and 8 riders. For groups of 6 or more similarly-sized riders, the one-but-last rider experiences the largest drag reduction. For smaller groups, it is the last rider that has the largest drag reduction. The reason is that the wake behind the riders widens with downstream position. Therefore, as an example, the last rider in a group of 3 benefits more than the last one in a group of 2 . The widening of the wake becomes less pronounced from about the $5^{\text {th }}$ position, and the beneficial effect of having a trailing rider in your wake then becomes comparatively more important. In addition, note that the leading cyclist in a team of three or more experiences a larger benefit $(3.1 \%)$ than in a team of two (2.6\%), due to the upstream disturbance of the flow (overpressure area) by the $3^{\text {rd }}$ rider that extends up to the position of the $1^{\text {st }}$ rider.

\section{Strategy in team time trials}

Many factors determine the outcome of a race. In regular races, the above-mentioned drag reductions for the leading cyclist are probably too low for this knowledge to lead to different racing behaviour. This is certainly the case for a chaotic peloton sprint. However, team time trials are much more organised than peloton sprints. In these races, a group of cyclists of the same team try to apply drafting in the best possible way to achieve the best possible team performance. During the race, the members of the team alternate to take the lead role.Apart from the alternating order, of course also body size and shape and position on the bike are important. A larger trailing rider will provide a larger drag reduction for a smaller rider in front of him, while the trailing rider himself will benefit less because of the smaller wake from the smaller leading rider. In addition, different cyclists also have different power curves. The best strategy and alternating sequence in team time trials can be determined based on the combination of aerodynamic drag simulations and power performance curves.

\footnotetext{
> FIG.3:

(a) Computational grid for two drafting cyclists (body of cyclist A) in time-trial position and with bicycle separation

distance $d=0.01 \mathrm{~m}$. The grid on the cyclist body and in the vertical centre plane is illustrated. (b) Detailed view of computational grid near upper body, head and helmet. Note the very high grid resolution at the body surface.
}
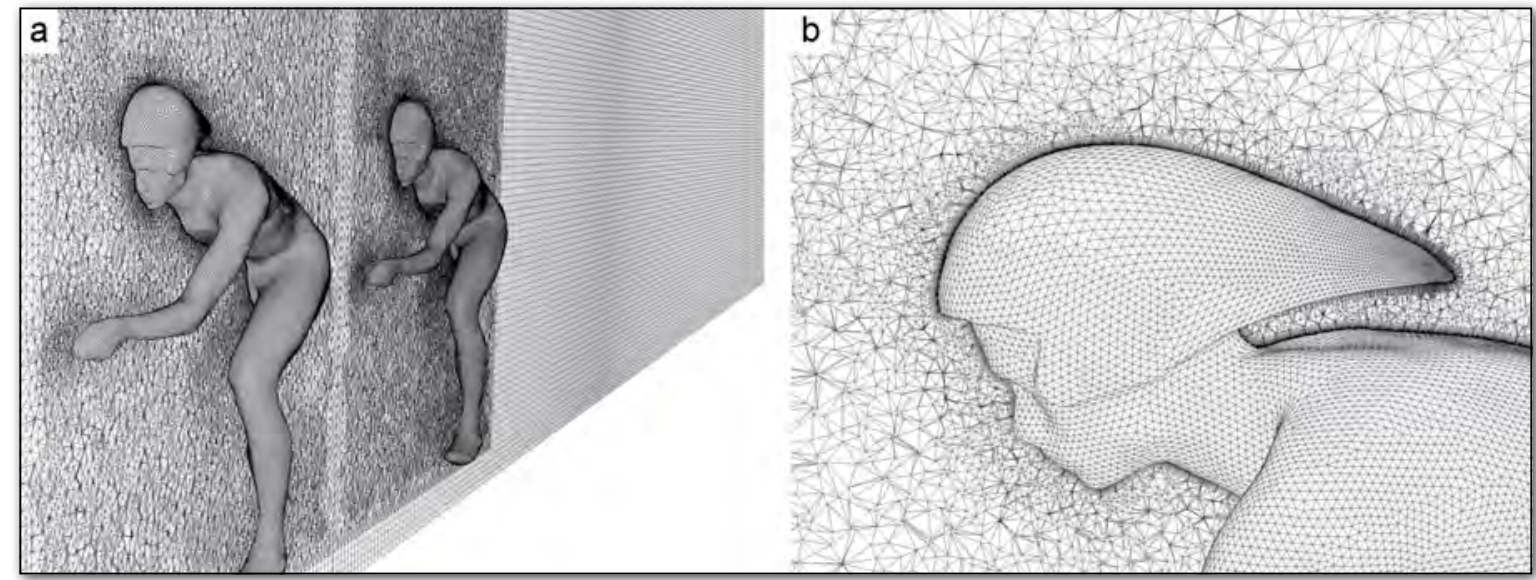


\section{Limitations}

The two main limitations of the study are, first, that all cyclists had identical body geometry and position on the bicycle, and second, that only static positions (i.e., no pedalling) were evaluated. The simulations were also performed for cyclists directly behind each other at a separation distance of only $0.01 \mathrm{~m}$. While this distance is unrealistically low when riding precisely behind each other, it should be noted that cyclists often ride much closer to each other, be it in a slightly staggered arrangement, with the front wheel of the trailing rider next to the back wheel of the leading rider. In such cases, the drag reductions might be even larger. Future work will include analysis of drag effects in such arrangements.

\section{Conclusions}

CFD simulations validated by wind-tunnel measurements show that drafting also benefits the leading rider, due to the presence of trailing riders in his wake. The drag reduction for the leading rider is up to $2.6 \%$ with one trailing rider and up to $3.1 \%$ with two or more trailing riders. The same effects imply that in a group of 6 or more similarly-sized riders, the position with the largest drag reduction is not the last, but the one-but-last. The difference in drag reduction between both positions is about $1 \%$. In top competitions, where winning or losing is often a matter of seconds, these drag reductions due to trailing riders are significant and can be decisive.

\section{About the Authors}

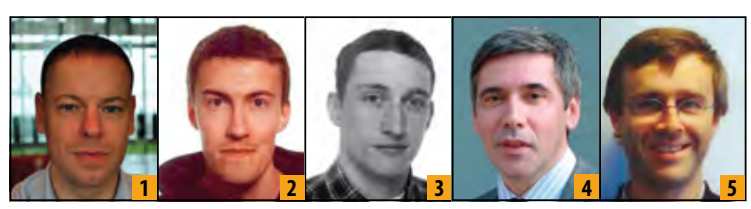

(1) Bert Blocken is full professor and chair of building physics at Eindhoven University of Technology, with specific expertise in (urban) aerodynamics. (2) Thijs Defraeye is a postdoctoral fellow of the FWO-Flanders in biosystems engineering at Leuven University in Belgium. (3) Erwin Koninckx is a postdoctoral researcher with a background in both engineering and biomedical kinesiology working at the Flemish Cycling Federation. (4) Jan Carmeliet is full professor and chair of building physics at ETH Zurich. (5) Peter Hespel is full professor in biomedical kinesiology at the Bakala Academy at Leuven University.

\section{Acknowledgements}

The hardware support of the Laboratory of the Unit Building Physics and Services for the CFD simulations and the high-quality assistance of the Dutch-German Wind Tunnel team (DNW) headed by Eddy Willemsen are gratefully acknowledged. Thijs Defraeye is a postdoctoral fellow of the Research Foundation - Flanders (FWO) and gratefully acknowledges its support.

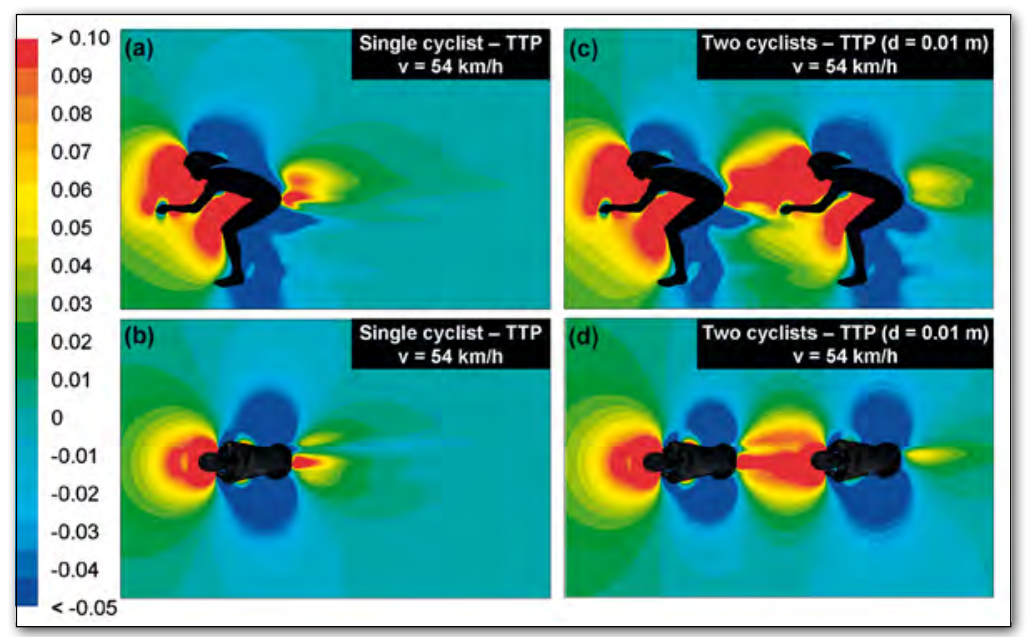

\section{References}

[1] D.G. Wilson, Bicycling science (2004). Third Edition, Cambridge, MA: MIT Press.

[2] C.R. Kyle, E.R. Burke, Improving the racing bicycle. Mech Eng 106(9), 34 (1984)

[3] R.A. Lukes, S.B. Chin, S.J. Haake, Sports Eng 8, 59 (2005)

[4] T. Defraeye, B. Blocken, E. Koninckx, P. Hespel, J. Carmeliet, JBiomech 43(7), 1262 (2010a).

[5] T. Defraeye, B. Blocken, E. Koninckx, P. Hespel, J. Carmeliet, J Biomech 43(12), 2281 (2010b).

[6] T. Olds, Eur J Appl Phys 77, 492 (1998).

[7] C.R. Kyle, Ergonomics 22(4), 387 (1979).

[8] S.D. McCole, K. Claney, J.-C Conte, R. Anderson, J.M. Hagberg, JAppl Phys 68(2), 748(1990).

[9] A. Iniguez-de-la-Torre, J. Iniguez, Eur J Phys 30, 1365 (2009).

[10] M. Casey, T. Wintergerste, Best Practice Guidelines (2000). ERCOFTAC Special Interest Group on "Quality and Trust in Industrial CFD", ERCOFTAC.

[11] J. Franke, A. Hellsten, H. Schlünzen, B. Carissimo, Best practice guideline for the CFD simulation of flows in the urban environment, COST Action 732: Quality assurance and improvement of microscale meteorological models, Hamburg, Germany (2007).

[12] Y.Tominaga, A. Mochida, R. Yoshie, H. Kataoka, T. Nozu, M. Yoshikawa, T. Shirasawa, JWind Eng Ind Aerodyn 96(10-11), 1749 (2008).

[13] B. Blocken, C. Gualtieri, Environ Modell Softw 33, 1 (2012).

[14] W.P. Jones, B.E. Launder, Int J Heat Mass Transfer 15, 301 (1972).

[15] M. Wolfshtein, Int J Heat Mass Transfer 12(3), 301 (1969).

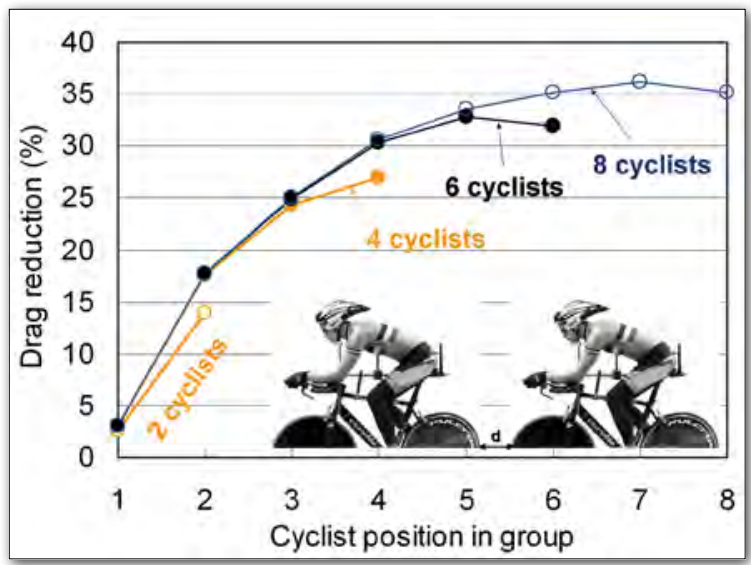

$\triangle F I G .4:$

Pressure coefficients $C_{p}$ in the vertical centre plane $(\mathrm{a}, \mathrm{c})$ and in a horizontal plane (b,d) at waist height for $(a, b)$ isolated (single) cyclist and (c,d) two drafting cyclists in time-trial position (TTP) with bicycle separation distance $d=0.01 \mathrm{~m}$. The cycling speed is $54 \mathrm{~km} / \mathrm{h}$.

4 FIG. 5: Numerically simulated drag reduction in groups of $2,4,6$ and 8 cyclists, in time-trial position with bicycle separation distance $d=0.01 \mathrm{~m}$. 